\title{
Zum Luciantext der Genesis.
}

\author{
Von Pfarrer Johannes Dahse in Freirachdorf (Westerwald).
}

Vor zwei Jahren habe ich an dieser Stelle die Septuagintahandschriften der Genesis zu gruppieren versucht und die vier Gruppen ackmosxc ${ }_{2}$, egj, $\mathrm{f}\left(\mathrm{i}^{\mathrm{a}}\right) \mathrm{r}$ und dnpt unterschieden. Meine Gruppierung der Handschriften hat nunmehr die Billigung von E. HAUTSCH in dem ersten Heft der Mitteilungen des Septuaginta-Unternehmens der KGdW zu Göttingen gefunden, nicht aber ist er mit meinen Ausführungen über die Herkunft der verschiedenen Textgestaltungen einverstanden; jenes erste Heft, betitelt: Der Lukiantext des Oktateuch, wendet sich gegen meine Auffassungen von dem Genesistext LUCIANs. Wohl stimmt HaUTSCH darin mir bei, daß wir in der Genesis in den Handschriften 19 und 108 LuCIAN nicht vor uns haben; nicht zustimmen aber kann er meiner Ansicht, daß uns LUCIAN in der Gruppe fir in der Genesis erhalten ist, und er sucht meinen dafür in ZAW 1908 geführten Beweis zu entkräften. Ich glaube aber, HAUTSCH unterschätzt die von mir angeführten Gründe, und aufgefallen ist $\mathrm{mir}$, daß er sie zum Teil ungenau und überhaupt nicht einmal alle in seiner Gegenschrift anführt. Zunächst kommt das Verhältnis der Handschrift $56(=\mathrm{i})$ zu 198293 I08 inbetracht. Darüber schreibt Hautsch S. 4 ,Daß Cod. $\mathrm{i}(56)$ in den Büchern Regn. oft mit den L-Hss. I9 82 93, 108 zusammengeht, beweist noch nicht, daß er in der Genesis den Luciantext bieten müsse". Nach HaUTSCH' Worten könnte es scheinen, als ob es sich bloß um die Textlesarten von 56 handelt; ich habe aber doch in ZAW I908 S. I9 ausdruicklich auch das angeführt, daf die Randlesarten von 56 in der Genesis derselben Art sind wie in den Büchern Regn., daf diese Randlesarten also eine zweite Handschrift voraussetzen, die in den Büchern Regn. den Text von 19 8293 108, in der Genesis den von 53 I 29 bot. Selbstverständlich können Handschriften, die mehrere Bücher enthalten, in den verschiedenen Büchern einen verschiedenen Textcharakter haben, aber hier liegt die Sachlage doch etwas anders. Wenn in den Büchern Regn. der lucianische Text häufig durch die Gruppe $19 \quad 56 \quad 82 \quad 93 \quad 108 \quad 246$ gegeben wird, und in der Genesis von diesen Handschriften vier nicht inbetracht kommen 
(93 nicht, da diese Handschrift erst mit dem Buche Ruth beginnt, 82 nicht, da sie, wie von mir nachgewiesen, in der Genesis hexaplarischen Text gibt, und 19 und 108 nicht, worüber zwišchen HAUTSCH und mir Úbereinstimmung herrscht), so wird doch zunächst die Vermutung erlaubt sein, daß die Gruppe, zu der 56 in der Genesis gehört und die eine besonders auffallende Textgestalt hat, uns da, wo 19 und 108 versagen, Lucian erhalten haben könnte. Diese Vermutung gewinnt an Wahrscheinlichkeit, wenn wir sehen, daß 56 auch in den Makkabäerbüchern Beziehungen zu LUCIAN hat, indem nach G. SCHMidT ZAW r897 S. 252 die Handschrift 56 ein Mittelglied zwischen dem Vulgärtext und dem LUCIANs bildet, übrigens neben 55, weswegen ich mit Absicht und mit Recht letzteren Cod. in ZAW 1908 S. I9 neben 56 und 129 angeführt habe und diese meine Angabe von HaUTSCH nicht mit einem ,so!" versehen zu werden brauchte. Noch mehr gewinnt aber meine Vermutung dadurch an Gewicht, daß die Sonderlesarten von fir in der Genesis denselben Charakter tragen, wie die Sonderlesarten LUCIANs in den zugestandenermaßen zu seiner Rezension gehörenden Handschriften. Neuerdings hat uns PROCKSCH in seiner Schrift: „Studien zur Geschichte der Septuaginta. Die Propheten" ein Bild von der Arbeitsweise LuCLans entworfen. Seine Charakteristik LưCIANs stimmt aber mit dem überein, was ich ZAW I908 S. 14 und 166 , als Eigenarten der Gruppe fir angegeben habe. Nach PROCKSCH ist am meisten für LUCIANs Arbeitsweise charakteristisch seine interpretatorische Tätigkeit, seine Revisionsarbeit inbetreff lexikalischer, stilistischer und grammatischer Dinge. Ebensolche Interpretamente, die den Sinn präzisieren und stilisieren, wie sie PROCKSCH S. 82 anführt, haben wir aber in der Genesis gerade bei Gruppe fir und bei. dieser Gruppe in viel größerem Maße als.bei egj, deren Autor vor umfassenderen Änderungen eine Scheu hat. Vor fünf Jahren habe ich mir folgende Liste der Eigentümlichkeiten von $56 \quad 129$ aufgestellt, die aber auf Vollständigkeit ganz und gar keinen Anspruch macht, sondern nur ein Bild von unserm Revisor geben soll:

I. Erklärende Zusätze:

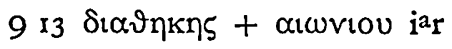

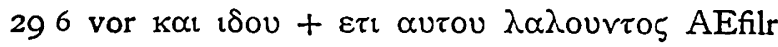

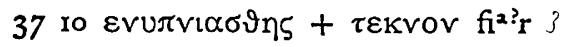

$3722 \lambda \alpha \kappa k \omega v+\tau o u \tau \omega v \mathrm{D}($ ?)bfirw

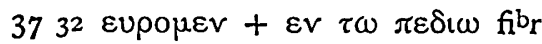

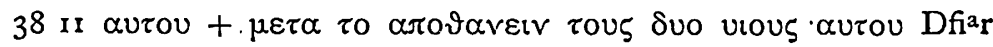

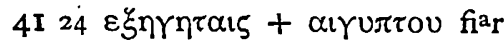


$4 I 37 \dot{p} \eta \mu \alpha \tau \alpha+\tau \alpha u \tau \alpha$ fiar

$4 \mathrm{I} 48 \alpha v \tau \eta 5$ +. $\omega v \mathrm{Afi}^{2}{ }^{\mathrm{r}} \mathrm{r}$

$4827 \sigma \varphi \circ \delta \rho \alpha+$ or $\delta \omega \delta \varepsilon \kappa \alpha f^{2}$ ?

Besonders auch

a) Hinzufügung der Kopula

2527 I $\alpha \omega \beta \delta \varepsilon+\eta \vee$ Abfhiorwy

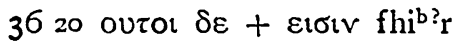

$3825 \tau i v 05+\varepsilon \sigma \tau i v$ fiar

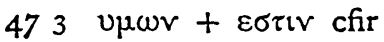

b) Hinzufügung von Subj. oder Obj. beim Verbum des Sagens

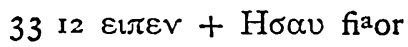

$3716+\alpha u \tau \omega$ Lir

$397+\alpha v \tau \omega$ fialmr

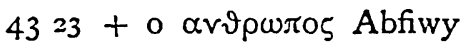

47 II $\sigma u v \varepsilon \tau \alpha \xi \varepsilon v+\alpha u \tau \omega \mathrm{f}^{2} \cdot \mathrm{r}$

2. Lexikalische Revision

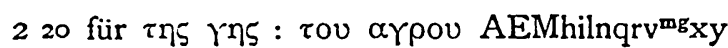

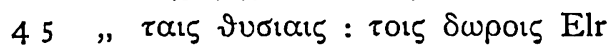

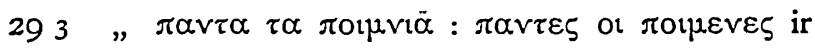

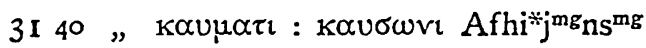

3720 „ о

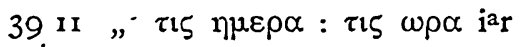

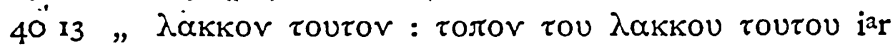

4316 "

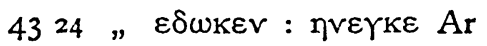

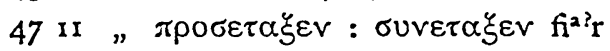

4814 \# $\varepsilon \pi \varepsilon \beta \alpha \lambda \varepsilon \vee$ : $\varepsilon \pi \varepsilon \vartheta \eta \kappa \varepsilon \vee f^{a}{ }^{3} r$

3. Revision hinsichtlich der Verbalformen

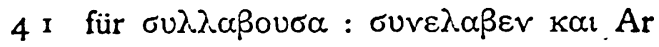

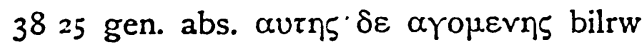

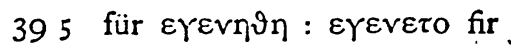

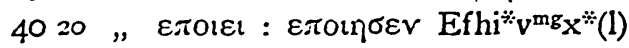

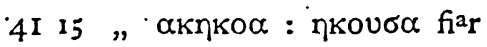

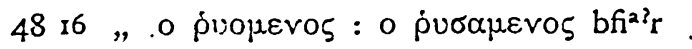

4. Revision hinsichtlich der Partikel

3821 or $\delta \varepsilon$ statt $\mathrm{kal}$ fir

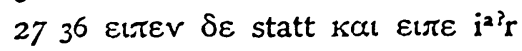

4 II $\varepsilon \pi$ statt a $\alpha$ Aiy

$31=\varepsilon \chi \vartheta \varepsilon 5$ statt $\chi \vartheta \varepsilon 5$. AEMaiors 


\section{Umstellungen}

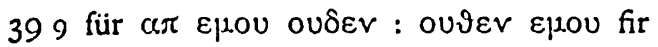

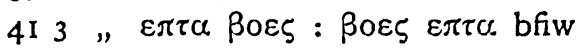

\section{Stilistisches}

38 30 für $\varepsilon \varphi \omega \eta \eta$ : $\varepsilon x \omega r$ fiar

3920 " $\varepsilon v \omega:$ ou fi

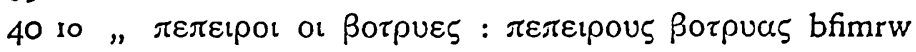

Ich denke mit dieser Liste genügend Material zur Beurteilung der Revision, die uns in fir in interpretatorischer Hinsicht ${ }^{x}$ vorliegt, geliefert $\mathrm{zu}$ haben, und glaube mit Recht auf Grund dieser sprachlichen Eigenarten fir für LUCIAN beanspruchen zu können. Wenn HAUTSCH S. 5 bemängelt, $\mathrm{da} ß$ ich die Schilderung THECDORETs über LuCIANs Arbeitsweise ZAW $1908 \mathrm{~S}$. I6I f. zum Beweise für meine Auffassung herangezogen habe, so geht doch schon daraus, daß ich sie erst in meinem zweiten Aufsatze heranziehe, hervor, daß es mir nur darauf ankam zu zeigen, daß die in fir vorliegende Revision jener Charakteristik nicht widerspricht. Auf sie Rücksicht zu nehmen, war meine Pflicht; meine positiven Beweise finden sich in "Textkr. Studien I“. Ich kann heute zum Beweise für lucianische Herkunft von fir weiter darauf hinweisen, dab die Handschrift $f(=53)$, die 1439 geschrieben ist, im Jahre 1740 von Konstantinopel nach Paris gekommen ist, und $\mathrm{da} ß$ sich für eine andere $z u$ unserer Gruppe gehörende Handschrift vielleicht noch der Beweis führen läßt, daß sie aus Syrien nach Europa gekommen ist, wie ich das schon ZAW 1908 S. 164 angedeutet habe.

Nun behauptet HAUTSCH auf Veranlassung von RAHLFS, dab es noch gar nicht fest stehe, ob dịe äthiopische Übersetzung, deren so häufige Übereinstimmung mit fir ich zum Beweise für syrische Herkunft dieser Textform angefüht, da Äthiopien durch syrische Missionare sein Christentum und seine Bibel erhalten habe, wirklich L-text enthalte. Die Polemik gegen meine Auffassung hängt zusammen mit RAHLFS' Ansicht von der Herkunft von $B$, welche Handschrift nach ihm durch das Fehlen der Makkabäerbücher, gemäß dem Kanon des AtHaNAsIus, als ägyptisch dokumentiert sei; B habe aber Beziehungen zum Äthiopen; durch ägyptische Einflüsse erkläre sich auch das Fehlen der Makkabäerbücher in der äthiopischen Übersetzung. Aber nun haben sich doch die Äthiopier inbetreff der'Pseudepigraphen gar nicht nach dem Kanon des ATHANASIUS gerichtet; sollten sie es nur bei den Büchern der Makkabäer getan haben?

I Auf das Verhāltnis zum Hebräer gehe ich hier nicht ein. 
Ist überhaupt das Fehlen der Makkabäer nur bei ATHANASIUS zu finden und ein genügender Beweis für ägyptische Herkunft? Dann müßten ja die beiden Handschriften IOS und w (= Athen, Bibl. Nat. 44), in denen nach SWETE die Makkabäerbücher fehlen, hesychianisch sein, und die Handschrift 58, die in der Genesis hexaplarischen Text hat und syrische Einflüsse zeigt, in der ebenfalls die Malkkabäer fehlen ${ }^{3}$, Beziehungen zu Ägypten haben! Wir sehen, das Fehlen der Makkabäerbücher bei B darf nicht zum Beweise für ägyptische Herkunft herangezogen werden; haben wir dasselbe doch auch gerade bei lucianischen und zu Syrien in Beziehung stehenden Texten. Somit beweist das Fehlen der Makkabäer in der äthiopischen Übersetzung gar nichts dafür, daß Äthiopien aus $\ddot{A}$ gypten seinen Bibeltext erhalten hat. Zur Begründung meiner These habe ich a. a. O. S. 163 darauf aufmerksam gemacht, daß sowohl der Äthiope wie auch Gruppe fir Beziehungen zu der als o ovpos vorkommenden Textgestalt haben. Von HAUTSCH wird dieser Nachweis ignoriert! Für meine Auffassung von der Herkunft des äthiopischen Christentums aus Syrien kann ich mich jetzt auf den Artikel „AbessinienÄthiopien in der Kirchengeschichte" in RGG I Spalte Ioof. berufen, und Herr Prof. LitTManN hatte die Freundlichkeit, mir auf meine Anfrage brieflich mitzuteilen, daß die regeren Beziehungen zwischen Abessinien

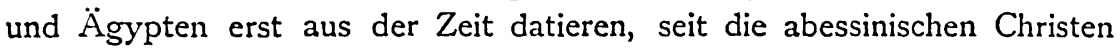
vom roten Meere abgeschnitten waren, und daß das äthiopische $A T$ von Leuten; die Aramäisch als Muttersprache redeten, aus dem Griechischen übersetzt sei.

In seiner Abhandlung kommt HAUTSCH nun zu dem Resultate, daß, abgesehen von der Genesis, in den übrigen Büchern des Pentateuch die Handschriften (44) 54 (59) $74 \quad 75 \quad 76$ I06 I34 den antiochenischen Kirchenvätern besonders nahe stehen und da $b$ in Josua innerhalb dieser Gruppe 5475 sich durch ein besonders nahes Verhältnis zum Text des Thdt auszeichnen. Ich selbst habe schon ZAW rgo8 S. I4 darauf aufmerksam gemacht, daß zu meinem .LuCIAN gerade auch 59 75. Beziehungen haben, habe aber auch darauf hingewiesen, dab wir $75(=\dot{n})$ wiedertreffen bei meiner Gruppe dnpt ${ }^{2}$. Nun behauptet HaUTsCH S. 14, daf in der Genesis sehr häufig die Handschriften defnopstc ${ }_{2}$ mit den Lesarten der antiochenischen Väter zusammengehen, macht aber gleich die Einschränkung, dab kaum in einem Falle alle allein mit den $\mathrm{KV}$ übereinstimmen. Letzteres ist kein Wunder, da die von HAUTSCH auf-

I Sie fehlen auch in 248 .

2 Ebenso 54 cf. ZAW $190 \mathrm{~S}$ S. 7. 
gezählten Handschriften zu vier verschiedenen Gruppen gehören: e zu egj, $\mathrm{f} z \mathrm{fu}$ fir, osc $\mathrm{C}_{2}$ zur Hexapla und Gruppe dnpt! Warum wird es nun aber von HAUTSCH nicht erwähnt, daß ich es a.a. O. S. 20 mit 20 Stellen belegt habe, daß Chr dem Texttypus der Lektionarien folgt, der zu meiner Gruppe dnpt die engsten Beziehungen hat? Und damit komme ich zu einer Frage, die gerade im jetzigen Zustande der Rezensionenfrage gelöst werden muß: Steht es überhaupt fest, dalj die Anticchener immer den ursprünglichen Text LUCIANs gebrauchen? Hat nicht E. NESTLE schon Sept. Studien IV S. I 3 darauf hingewiesen, daB THEOdoret in den Propheten den durch die aldina gegebenen Text zitiert und wird dieser jetzt nicht außer von FAULHABER und mir auch von PROCKSCH S. 64 als hexaplarisch angesehen und von dem lucianischen Text in den Propheten unterschieden? Wenn HAUTSCH mir entgegenhält, da@ die äthiopische Übersetzung nicht zum Beweise herangezogen werden könne, „da es noch gar nicht feststeht, ob sie wirklich L-Text enthält", so kann ich ihm mit größerem Rechte entgegnen: $D a$ es bewiesen ist, daß die Antiochener auch andere als Lucians Texte benutzen, so ist es falsch, lediglich auf ihr Zeugnis hin LUCIANs Text bestimmen zu wollen! Dafj man nicht immer auf sicheren Wegen geht, wenn man auf den Zitaten der Antiochener seine Ansicht aufbaut, muß uns HAUTSCH selbst bestätigen. Denn während er S. 5 behauptet: $\mathrm{Da}$ sich das Zeugnis des Hieronymus für die übrigen historischen Bücher des AT bewahrheitet hat, so wird man gut tun, auch bei der Bestimmung des L-Textes des Oktateuch von dem Verhältnis der Hss zu den antiochenischen Vätern auszugehen, muß er S. 27 bekennen, daß das Urteil des HiERonymus doch nicht in dem Maße gelte, wie es gewöhnlich gewertet wird; die Lukianrezension sei bereits innerhalb der antiochenischen Schule durch die hesychianische Rezension beeinträchtigt! Meines Erachtens ist nicht von den Zitaten der Antiochener auszugehen, wenn man Lucians Rezension, soweit sie noch unbekannt, finden will, sondern zuerst hat man auf Grund der Septuagintahandschriften, wie ich es in meinen „Textkr. Studien" versucht habe, die verschiedenen Texttypen festzustellen, und dann $z u$ prüfen, $z u$ welcher der von den Alten genannten Rezensionen die noch heute erkennbaren Typen in Beziehung gesetzt werden können.

Was nun die Gruppe dnpt anlangt, so' habe ich ZAW I908 S. 2I Gründe dafür angeführt, daß ihr Text aller Wahrscheinlichkeit nach aus dem Bereiche von Antiochien-Konstantinopel stamme. Aber in dieser Gruppe liegt uns nicht der reine Lucian vor. Schon DE LAGARDE sagt von 44, daß diese Handschrift nicht auf gleicher Stufe 
stehe mit I9 8293 108, rechnet sie aber doch zur weiteren Verwandtschaft LuCians. Holmies bestimmt sie als tetraplarisch und läbt den LUCIAN die Tetrapla benutzen. ProckSCH rechnet die verwandte Handschrift $106 \mathrm{zu}$ seiner vorhexaplarischen Gruppe (II), hat also für die Propheten altes Gut in ihr erkannt, wie ich für die Genesis. Und wenn ich für die Genesisperikopen darauf aufmerksam gemacht habe, daß der Text der alten Triodien zur Gruppe dnpt gehört, so hatte A. ZiLlessen die Freundlichkeit, mir mitzuteilen, daß in den Jes. Perikopen die Lesarten des Triodiums von 1599 vor allem die von 62147 und von 106 seien! Also auch in den Propheten bietet 106 und Gen. einen im Bezirk der Kirche von Konstantinopel offiziellen Text, der hexaplarische und lucianische Elemente ${ }^{x}$ in sich trägt und von dem reinen LUCIAN $22 \quad 36 \quad 48 \quad 5$ I $93 \quad 144308$ unterschieden werden muß. Wie in der neutestamentlichen Textkritik haben wir auch in der Septuagintaforschung mit zwei Rezensionen, die von Syrien bis Konstantinopel in Gebrauch waren, $z$ u rechnen; wie sie sich $z u$ einander verhalten, müssen spätere Untersuchungen klarstellen.

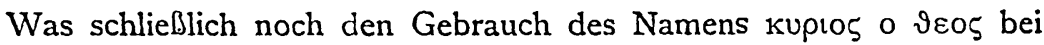
fir in der Genesis anlangt, den ich als lucianisch ansehe, so werde ich mich mit dem Widerspruch von HAUTSCH gegen meine Auffassung in einer größeren Abhandlung auseinandersetzen und in der Deutung der

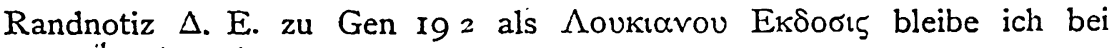
meiner Auffassung, bis mir von HAUTSCH eine bessere Deutung vorgeschlagen wird.

I Vgl. hierzu Procksch S. 76. 